\title{
Syntheses and In-vitro Evaluation of \\ Tetrahydroaminoacridine (THA) Based Analogues as High Affinity Choline Transporter (HAChT) Imaging Probe
}

\author{
${ }^{1}$ Mohammad Anwar-Ul Azim, ${ }^{2}$ Takashi Kozaka, ${ }^{2}$ Izumi Uno, ${ }^{2}$ Daisuke Miwa, \\ ${ }^{2}$ Yoji Kitamura, ${ }^{3}$ Kazuma Ogawa, ${ }^{2}$ Kahuhiro Shiba \\ ${ }^{1}$ National Institute of Nuclear Medicine and Allied Sciences, BAEC, BSMMU, Shahbagh, Dhaka. \\ ${ }^{2}$ Division of Tracer Kinetics, Advanced Science Research Center, Kanazawa University, Japan. \\ ${ }^{3}$ Institute of Medical, Pharmaceutical and Health Sciences, Kanazawa University, Japan.
}

Correspondence: Dr. Mohammad Anwar-Ul Azim, Senior Scientific Officer, National Institute of Nuclear Medicine \& Allied Sciences (NINMAS), Bangladesh Atomic Energy Commission (BAEC), BSM Medical University Campus, Block-D, $8^{\text {th }}$ Floor, Shahbagh, Dhaka-1000. E-mail: anwarri79@gmail.com

\begin{abstract}
Introduction: In cholinergic neurons, high affinity choline uptake (HACU) by the high affinity choline transporter (HAChT) is a rate-limiting and regulatory step for the synthesis of Acetylcholine (Ach).Thus, HAChT appear to be a relatively specific presynaptic marker for cholinergic neurons in Alzheimer's disease.

Objectives: The principle objective of the study is to check the affinity of tetrahydroaminoacridine (THA) derivatives for HAChT. Another objective of the research work is to clarify whether the hemicholinium-3 (ChT inhibitor) and HACU enhancer molecules share the same binding sites or not.

Materials and Methods: The inhibition activities of tacrine, the 2,3-dimethylfuran derivative of tacrine (DMTA) and their corresponding 2-oxo-1-pyrrolidineacetyl derivatives, namely PTAA and MKC-231 were measured by displacement of a typical HAChT antagonist $\left[{ }^{3} \mathrm{H}\right] \mathrm{HC}-3$ in rat cerebral membrane. The percentage of inhibition against the binding of $\left[{ }^{3} \mathrm{H}\right] \mathrm{HC}-3$ to HAChT were calculated using GraphPad Prism v4 software.
\end{abstract}

Results: Hemicholinium-3 showed affinity for HAChT $\left(\mathrm{IC}_{50}=\right.$ $20 \mathrm{nM}$ ) in the in vitro binding assay. A very insignificant inhibition activity $\left(\mathrm{IC}_{50}=1000 \mathrm{nM}\right)$ of Tacrine was revealed. The newly synthesized tacrine derivatives, DMTA and PTAA did not show any affinity for HAChT. Although MKC-231 was reported to enhance cholinergic activity at synaptic terminals, it did not show any affinity for the HAChT in $\left[{ }^{3} \mathrm{H}\right] \mathrm{HC}-3$ binding assay.

Conclusion: In vitro $\left[{ }^{3} \mathrm{H}\right] \mathrm{HC}-3$ binding assay revealed no affinity of MKC-231, tacrine and its corresponding2-oxo-1pyrrolidineacetate derivative towards HAChT. So, it is worthy to develop radiolabeled $\mathrm{HC}-3$ derivatives with high affinity for HAChT, which can diffuse the BBB, to enable the in vivo investigation of HACU system.

Key Words: Tacrine, High Affinity Choline uptake, MKC-231, Cholinergic Neurotransmission.

\section{INTRODUCTION}

The high-affinity choline uptake system located in peripheral and central cholinergic nerve terminals plays a regulating and rate-limiting role in the intraneuronal synthesis of acetylcholine (ACh) $(1,2)$. Choline is transferred into the cell by the high-affinity choline transporter where it reacts with acetyl $\mathrm{CoA}$ in the presence of the enzyme choline acetyltranferase (ChAT) to form acetylcholine. Brain synaptosome studies demonstrate two carrier-mediated transport systems for choline uptake $(3,4)$. At high concentrations, choline is transported primarily by a low-affinity $\mathrm{Na}^{+}$-independent system that is inhibited by hemicholinium-3 (HC-3) (5) with a high $K_{\mathrm{i}}$ of approximately $50 \mu \mathrm{M}$. This system is thought to be ubiquitously present in cells and to be required for phosphatidylcholine synthesis. At low concentrations choline is transported by a high-affinity, $\mathrm{Na}^{+}$-dependent system that is inhibited by HC-3 with a low $K_{\mathrm{i}}$ of $10-100 \mathrm{nM}$. The high-affinity system is supposed to be present specifically in cholinergic neurons, because a substantial proportion of choline is converted to acetylcholine only when taken up through the high-affinity system $(3,4)$. The proposal that the high-affinity choline transport system is unique to cholinergic neurons is supported by the selective loss of the highaffinity choline uptake following depletion of cholinergic terminals in a variety of denervation studies (4). Choline uptake is generally believed to be the rate-limiting step in acetylcholine synthesis $(3,4)$. In addition, the high-affinity choline uptake is regulated by neuronal activity 
activity also regulate acetylcholine synthesis. In Alzheimer's disease, cholinergic neurons selectively degenerate. Consistent with the above hypothesis, the high-affinity choline transporter is reduced in Alzheimer's disease (7).

Pathogenesis of AD is highly complex. Researchers around the globe have proposed several hypotheses for explaining the mechanism of $\mathrm{AD}$ development. Among the hypotheses, cholinergic hypothesis has been widely approved and the most characteristic abnormality associated with $\mathrm{AD}$ is the decline of cholinergic neurotransmission. Deficiency in high affinity choline uptake (HACU) by the high affinity choline transporter (HAChT) and the loss of vesicular acetylcholine transporter (VAChT), are two characteristic neurochemical changes in AD. High affinity choline uptake transporter can serve as a useful marker of the functional status of the cholinergic presynaptic terminals and is known to be associated with a variety of diseases, such as Parkinson's disease, Alzheimer's disease, coronary heart diseases, tumor cancers $(8,9)$. Thus, choline transporter systems provide attractive targets for the development of PET biomarkers to probe brain, heart, and cancer diseases.

Tacrine (9-amino-1,2,3,4-tetrahydroacrydine, THA, commercial name: Cognix), is a well known acetylcholinesterase (AChE) inhibitor, which produces clinically significant improvement in some cognitive deficits observed in $\mathrm{AD}$ patients by increasing the synaptic availability of Ach by inhibiting the AChEmediated degradation of ACh to choline and acetate. But efficacies of this drug are limited and commonly associated with gastrointestinal side effects during long treatment period (10). Besides this, there is a deficiency of choline uptake in $\mathrm{AD}$ patients. Hence, activation of the presynaptic cholinergic function by improving the reduced HACU is another better approach for the treatment of $\mathrm{AD}$.

Although, THA has no effect as HACU enhancer, THA contained 4-aminopyridine (4 - AP) as a partial structure, which is already known to increase HACU and 1-nitrogen atom of 4-AP, also corresponds to the quaternary amine of $\mathrm{ACh}$ (Figure 1).In 1995, Chaki, H., et al showed acylation of THA leads to the synthesis of novel choline uptake enhancers which improve the reduced HACU and also that some of the structural features of $\mathrm{N}$-acyl derivatives of THA resembles acetylcholine. But due to this structural modification THA lose its activity as AChE inhibitors (11).
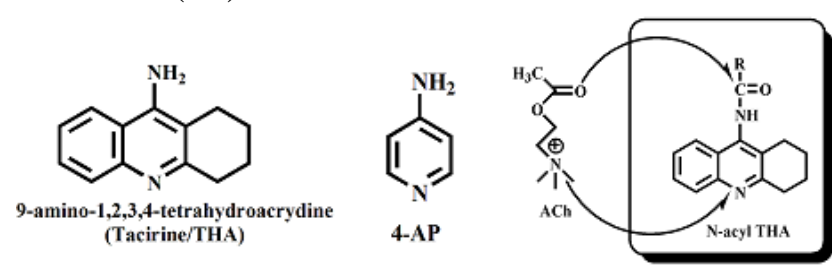

Figure 1. Chemical structure of THA, 4-AP, Ach and N-acyl THA Taking into the consideration, this special characteristic feature of $\mathrm{N}$-acyl derivatives of THA as HACU enhancer and the role of HAChT to enhance HACU, we have synthesized tacrine, the 2,3-dimethylfuran derivative of tacrine (DMTA) and their corresponding 2-oxo-1pyrrolidineacetyl derivatives, namely PTAA and MKC-231 and evaluated these compounds through in vitro $\left[{ }^{3} \mathrm{H}\right]-\mathrm{Hem}-3$ binding assay. The objectives of the evaluation of these THA derivatives are as follows: (a) to check the affinity of MKC-231, Tacrine and the newly synthesized THA derivatives (DMTA, PTAA) for HAChT, (b) to find out the possible explanation of mode of action (MOA) of HACU enhancer, and (c) to make a possible clarification of the fact that whether thehemicholinium-3 (ChT inhibitor) and HACU enhancer molecules share the same binding sites or not.

\section{MATERIALS AND METHODS}

Chemical Syntheses: The syntheses of Tacrine, the 2,3-dimethylfuran derivative of Tacrine (DMTA) and their corresponding 2-oxo-1pyrrolidineacetyl derivatives, namely PTAA and MKC-231, were accomplished with the following schemes: 
Scheme 1: Synthesis of tacrine (THA) and 2-oxo-1pyrrolidineacetyl derivative of tacrine, namely PTAA (Figure 2).

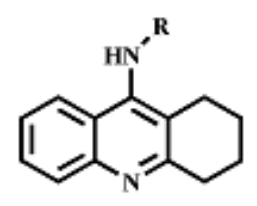

\begin{tabular}{|l|l|}
\hline Compounds & $R$ \\
\hline Tacrine (THA) & H \\
\hline PTAA &
\end{tabular}

Figure 2. Chemical structure of Tacrine and PTAA

Scheme 2: Replacement of the phenyl ring on the left part of the tricyclic structure of tacrine with a furan ring and synthesis of it's 2-oxo-1pyrrolidineacetyl derivative (Figure 3)<smiles>[Y]Nc1c2c(nc3oc([R])c(Br)c13)CCCC2</smiles>

\begin{tabular}{|l|c|c|c|}
\hline Compounds & $\mathbf{R}_{\mathbf{1}}$ & $\mathbf{R}_{\mathbf{2}}$ & $\mathbf{R}$ \\
\hline DMTA & $\mathrm{Me}$ & $\mathrm{Me}$ & $\mathrm{H}$ \\
\hline MKC-231 & $\mathrm{Me}$ & $\mathrm{Me}$ & \\
& & & \\
\hline
\end{tabular}

Figure 3. Chemical structure of DMTA and MKC-231

\section{Preparation of rat cerebral and liver membranes}

Animal experiments were performed in compliance with the guidelines for the care and use of Laboratory Animals at the Takara-machi Campus of Kanazawa University. Brain homogenate of rats were prepared with the slight modification of previously described protocol (12). Sprague-Dawley rats (8 weeks, male, 250-300 g) cerebrum or liver were homogenized in ice-cold $0.32 \mathrm{M}$ sucrose with a Teflon-glass homogenizer. The homogenate was centrifuged at $1000 \mathrm{~g}$ at $4{ }^{\circ} \mathrm{C}$ for $10 \mathrm{~min}$. The resulting precipitate was removed and the supernatant was centrifuged at $20,000 \mathrm{~g}$ at $4{ }^{\circ} \mathrm{C}$ for $20 \mathrm{~min}$. This crude mitochondrial pellet was resuspended in 20 vols. of ice-cold distilled water and dispersed with the Teflon homogenizer with 1000 rpm and the homogenate centrifuged at $8000 \mathrm{~g}$ run for $20 \mathrm{~min}$. The supernatant and buffy coat were collected and pelleted at 48,000 $\mathrm{g}$ for $20 \mathrm{~min}$. The pellet was washed 4 times in 20 vols. of $50 \mathrm{mM}$ Glycylglycine buffer ( $\mathrm{pH}$ 7.8) containing $200 \mathrm{mM}$ $\mathrm{NaCl}$ by resuspending via Teflon homogenizer. Then the homogenate was centrifuged at 55,000 $\mathrm{g}$ for about $15 \mathrm{~min}$ and the resulting pellet was again resuspended in $50 \mathrm{mM}$ Glycylglycine buffer $(\mathrm{pH}$ 7.8) containing $200 \mathrm{mM} \mathrm{NaCl}$ with a Teflon homogenizer (Figure 4).

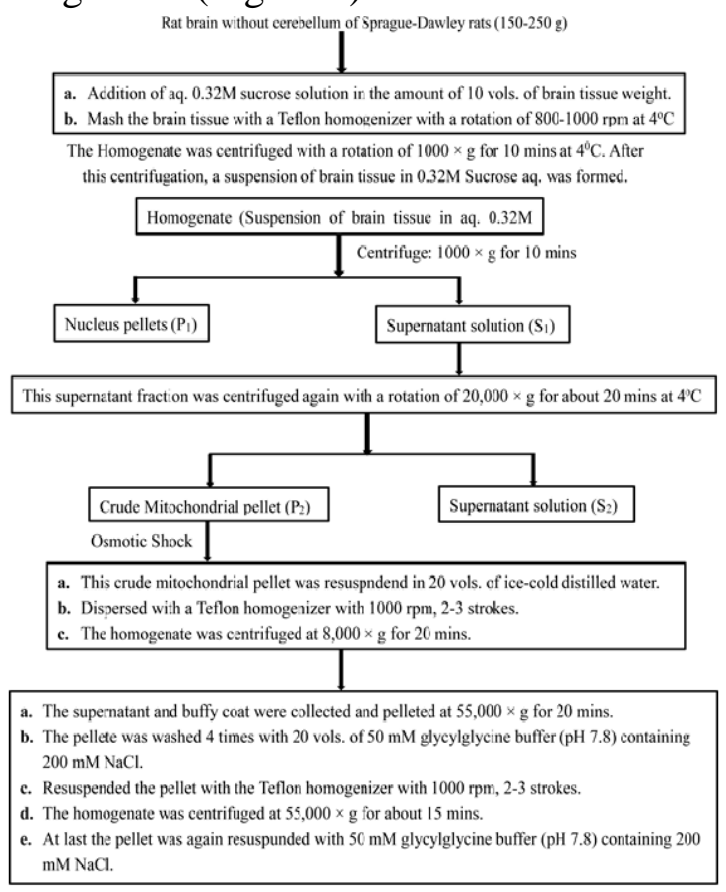

Figure 4. Flow chart for the homogenate reparation of rat cerebral and liver membranes.

\section{In vitro HAChT $\left[{ }^{3} \mathrm{H}\right]$ Hemicholinium--3 binding assay}

$\left[{ }^{3} \mathrm{H}\right]$ Hemicholinium-3 $\left(K_{\mathrm{d}}=19.1 \mathrm{nM}\right)(20)$ was used as a specific radioligand for the HAChT receptor. Rat cerebral membranes were added to each icecold assay tube containing $\left[{ }^{3} \mathrm{H}\right] \mathrm{HC}-3$ and the displacing ligands at various concentrations $(1.0 \times$ $\left.10^{-9}-10^{-4}\right)$ in $50 \mathrm{mM}$ Glycylglycine buffer $(\mathrm{pH} 7.8)$ containing $200 \mathrm{mM} \mathrm{NaCl}$ in quadruplicate. After the addition of tissues in an ice bath, each reaction mixture in the tube was incubated at $25^{\circ} \mathrm{C}$ for 30 min. The incubation was terminated by putting tubes into ice-cold water followed by immediate filtration using a cell harvester through glassmicrofiber filters (Whatman, GF/B), which were presoaked in $0.5 \%(\mathrm{v} / \mathrm{v})$ polyethyleneimine for one hour to reduce non-specific binding. The filters were washed with $50 \mathrm{mM}$ Tris-HCl buffer $(\mathrm{pH}=7.8)$, and the radioactivity was counted with the liquid scintillation counter (Aloka, LSC-5100). 


\section{RESULTS}

Chemistry: The cyclization of 2-Amino benzonitrile (1) and 2-Amino-4, 5-dimethyl-3-furancarbonitrile (2) with cyclohexanone by means of $\mathrm{ZnCl}_{2}$ in toluene, furnished tacrine (3) and DMTA (4), respectively (21)(Figure 5).
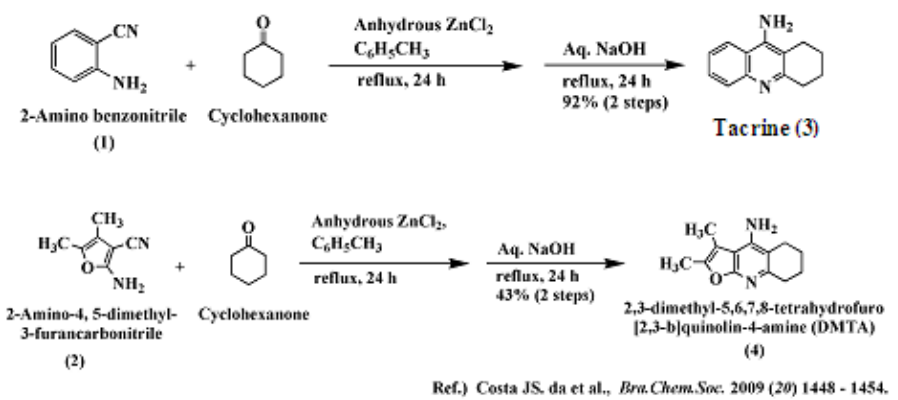

Figure 5. Scheme for the syntheses of tacrine and DMTA

The acylation of Tacrine (3) and DMTA (4) with methyl 2-oxo-1-pyrrolidineacetate furnished PTAA (5) and MKC-231 (6) with the yield of $79 \%$ and $65 \%$ respectively (Figure 6).

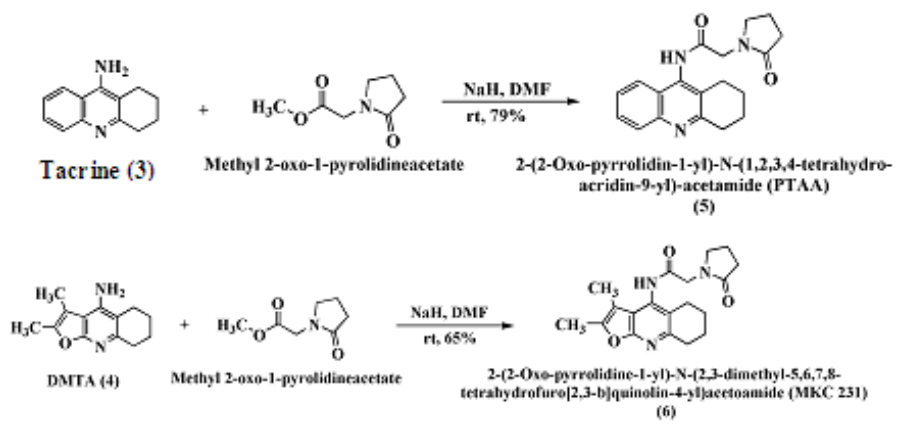

Figure 6. Scheme for the syntheses of PTAA and MKC-231

\section{In vitro HAChT $\left[^{3} \mathrm{H}\right]$ Hemicholinium-3 $\left(\left[^{3} \mathrm{H}\right] \mathrm{HC}-3\right)$ binding assay}

The inhibition activities of tacrine (THA), MKC-231 and the two newly synthesized tacrine derivatives, namely DMTA and PTAA were measured by displacement of a typical HAChT antagonist $\left[{ }^{3} \mathrm{H}\right] \mathrm{HC}-3$ (Dissociation constant: $K_{\mathrm{d}}=19.1 \mathrm{nM}$ ) (20) in rats cerebral membrane. The percent inhibition against the binding of $\left[{ }^{3} \mathrm{H}\right] \mathrm{HC}-3$ to HAChT were calculated using GraphPad Prism v4 software (Figure 7). HC-3 showed the highest affinity for $\mathrm{HAChT}\left(\mathrm{IC}_{50}=20 \mathrm{nM}\right)$. Tacrine (THA) showed very insignificant inhibition activity $\left(\mathrm{IC}_{50}=\right.$
$1000 \mathrm{nM}$ ).Both the 2, 3-dimethylfuran derivative of tacrine, DMTA and 2-oxo-1-pyrrolidineacetyl derivative of tacrine, PTAA showed no affinity for HAChT.MKC-231 also did not show any affinity for the HAChT in $\left[{ }^{3} \mathrm{H}\right] \mathrm{Hemicholinium}-3$ binding assay.

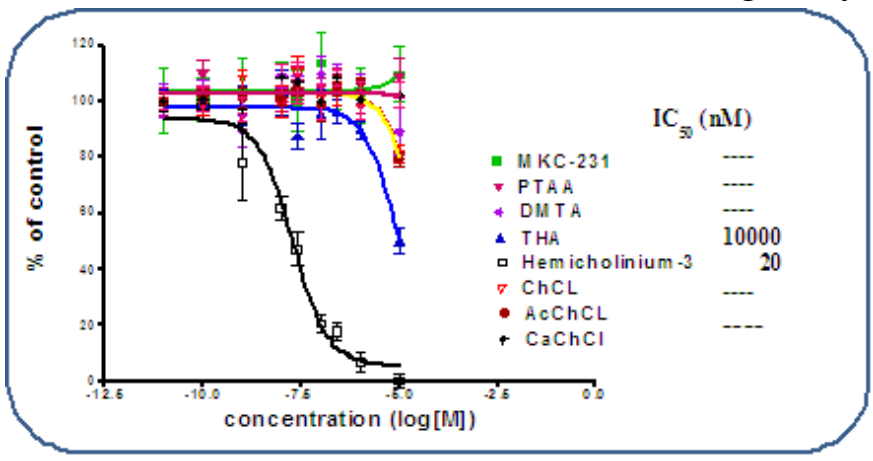

Figure 7. In vitro HAChT $\left[{ }^{3} \mathrm{H}\right]$ Hemicholinium-3 binding assay

\section{DISCUSSIONS}

High affinity choline uptake (HACU) system is located especially in presynaptic cholinergic nerve terminals. HACU by the HAChT is found to be a regulating and rate-limiting step in the synthesis of ACh. Hence, a ligand with a high affinity for HAChT would be an ideal marker for the study of the state of cholinergic presynaptic terminals.Hemicholinium-3 is an indirect acetylcholine antagonist, because it decreases the synthesis of acetylcholine by inhibiting the reuptake of choline by the high-affinity choline transporter (ChT). As HC-3 is a quaternary ammonium salt (Figure 8), it cannot cross BBB and radiolabeled quaternary ammonium $\mathrm{HC}-3$ derivatives cannot be used for in vivo visualization of the HACU transporter. MKC-231, 2-(2-Oxo-pyrrolidine1-yl)- $N$-(2,3-dimethyl-5,6,7,8-tetrahydrofuro [2, 3b]quinolin-4-yl) acetoamide, has been reported to enhance HACU in hippocampal synaptosomes in in vitro treatment and ameliorates learning deficits in AF64A-treated rats with a single oral administration (22). Although MKC-231 potentially enhances cholinergic activity at synaptic terminals and improves learning impairment observed in AF64Atreated rats and mice, no precise HACU 
enhancement mechanism has been elucidated. Acylation of THA, an AChE inhibitor, led to the synthesis of novel choline uptake enhancers, which improves the reduced HACUin AD. In the process THA lose its activity as AChE inhibitors (11).

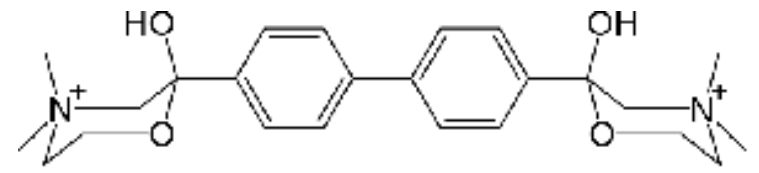

Figure 8. Chemical structure of Hemicholinium-3 (HC-3)

In the effort of developing HAChT imaging probe we have evaluated tacrine, the 2,3-dimethylfuran derivative of tacrine (DMTA) and their corresponding 2-oxo-1-pyrrolidineacetyl derivatives, namely PTAA and MKC-231 through in vitro $\left[{ }^{3} \mathrm{H}\right] \mathrm{Hemicholinium-3}\left(\left[{ }^{3} \mathrm{H}\right] \mathrm{HC}-3\right)$ binding assay to investigate their affinity for HAChT. From the in vitro binding assay, it was revealed that only hemicholinium-3 expressed high affinity for HAChT( $\left(\mathrm{IC}_{50}=20 \mathrm{nM}\right)$. Tacrine (THA) showed somedegree of inhibition activity $\left(\mathrm{IC}_{50}=1000 \mathrm{nM}\right)$, but it is very insignificant compared to the inhibition activity of hemicholinium - $3\left(\mathrm{IC}_{50}=20\right.$ $\mathrm{nM}$ ). Both the 2,3-dimethylfuran derivative of tacrine, DMTA and 2-oxo-1-pyrrolidineacetyl derivative of tacrine, PTAA showed no affinity for HAChT. Although, MKC-231, is known to enhance HACUand increase the synthesis and release of ACh in in vitro and in vivo studies (23), it did not show any affinity for the HAChT in $\left[{ }^{3} \mathrm{H}\right]$ Hemicholinium-3 binding assay. Other inhibitors like choline chloride $(\mathrm{ChCl})$, Acetylcholine chloride $(\mathrm{AChCl}), \quad$ Carbamylcholine chloride $(\mathrm{CaChCl})$ showed no binding affinity for high affinity choline transporter (ChT).

As no inhibitors (except Hem-3), MKC-231, THA and the synthesized THA derivatives did not exhibit any inhibition towards HAChT, so it is worthy to search for the new molecules having high affinity for choline transporter (HAChT) for the extensive study of HACU.

\section{CONCLUSION}

In a effort to develop tacrine and its 2-oxo-1pyrilidineacetyl derivatives as HAChT imaging probe, tacrine, DMTA (2, 3-dimethylfuran derivative of tacrine) and their corresponding 2oxo-1-pyrrolidineacetyl derivatives, namely PTAA and MKC-231, was synthesized and the binding affinities of these derivatives for HAChT were evaluated through in vitro HAChT $\left[{ }^{3} \mathrm{H}\right] \mathrm{HC}-3$ competitive binding assay. No affinity towards HAChT of the synthesized compounds was revealed from the In vitro binding assay.

Hemicholinium-3 is a competitive inhibitor of the HACU system and it is worthy to do further research works for the development of radiolabelled HC-3 derivatives with high affinity for HAChT, which can diffuse the BBB, to enable the in vivo investigation of HACU system. It is also worthy to do more extensive review to develop an effective in vitro $\mathrm{HAChT}\left[{ }^{3} \mathrm{H}\right] \mathrm{HC}-3$ competitive binding assay protocol and the protocol for the preparation of brain homogenate, used in the in vitro $\mathrm{HAChT}\left[{ }^{3} \mathrm{H}\right] \mathrm{HC}-3$ competitive binding assay.

\section{REFERENCES}

1. Smart LA. Synthesis and biological activity of a 2-bromoethylamine (mustard) derivative of hemicholinium-3 and hemicholinium-15. J Med. Chem1983; 26: 104-107.

2. Gilissen $\mathrm{C}$, de Groot $\mathrm{T}$, Bronfman F, van Leuven F, Verbruggen A. M, Bormans G. M. Evaluation of ${ }^{18} \mathrm{~F}-\mathrm{FA}-4$ and ${ }^{11} \mathrm{C}$-pipzA-4 asRadioligands for the In Vivo Evaluation of theHigh-Affinity Choline Uptake System. J Nucl. Med 2003;269-275.

3. Haga, T. Synthesis and release of $\left[{ }^{14} \mathrm{C}\right]$ acetylcholine in synaptosomes. J. Neurochem 


$$
\text { 1971;18:781-798. }
$$

4. Kuhar MJ, Murrin LC. Sodium-dependent, high-affinity choline uptake. J. Neurochem 1978;30:15-21.

5. Happe HK, Murrin LC. High-affinity choline transport sites: use of $\left[{ }^{3} \mathrm{H}\right]$ hemicholinium-3 as a quantitative marker. J. Neuro chem 1993; 60: 1191-1201.

6. Murrin LC, Kuhar MJ. Activation of highaffinity choline uptake in vitro by depolarizing agents. Mol. Pharmacol1976; 12:1082-1090.

7. Pascual J, Fontán A, Zarranz JJ, Berciano J, Flórez J, Pazos A. High-affinity choline uptake carrier in Alzheimer's disease: implications for the cholinergic hypothesis of dementia. Brain Res 1991; 552:170-174.

8. Hara T, Bansal A, DeGrado TR. Choline transporter as a novel target for molecular imaging of cancer. Molecular Imaging 2006; 5: 498-509.

9. Gao M, Miller MA, De Grado TR, Mock BH, Lopshire JC, Rosenberger JG, Dusa C, Das MK, Groh WJ, Zipes DP, Hutchins GD, Zheng $\mathrm{QH}$. Evaluation of $\left[{ }^{11} \mathrm{C}\right]$ hemicholinium-15 and $\left[{ }^{18} \mathrm{~F}\right]$ hemicholinium-15 as new potential PET tracers for the high-affinity choline uptake system in the heart. Bioorg. Med. Chem 2007; 15(3): 1289-1297.

10. Terry AV Jr, Buccafusco JJ. The cholinergic hypothesis of age and Alzheimer's disease related cognitive deficits: Recent challenges and their implications for novel drug development. J PharmacolExpTher2003; 306: 821-827

11. Chaki H, Yamabe H, Sugano M, Morita S, Bessho T, Tabata R, Saito KI, Egawa M, Tobe A, Morinaka Y. Design and synthesis of 4acylaminopyridine derivatives: Novel high affinity choline uptake enhancer $\mathrm{I}^{1)}$. Bioorganic and Medicinal Chemistry Letters
1995; 5 (14): 1489-1494.

12. Sandberg K, Coyle JT. Characterization of $\left[{ }^{3} \mathrm{H}\right]$ Hemicholinium-3 binding associated with Neuronal choline uptake sites in rat membranes. Brain research 1985; 348: 321330.

13. Quirion R. Characterization and autoradiographic distribution of Hemicholinium-3 high affinity choline uptake sites in mammalian brain. Synapse 1987; 1: 293-303.

14. Costa JS da, Pisoni DS, Silva CB da, Petzhold CL, Russowsky D, Ceschi MA. Lewis Acid Protonated Friedlander Condensation Reactions between Anthranilonitrile and Ketones for the Synthesis of Tacirine and its Analogues. J. Braz. Chem. Soc. 2009; 20 (9): 1448-1454.

15. Bessho $\mathrm{T}$, Takashina $\mathrm{K}$, Tabata R, Ohshima C, Chaki H, Yamabe H, Egawa M, Tobe A, Saito K. Effect of the novel high affinity choline uptake enhancer 2-(2-oxopyrrolidin-1yl)- $N$-(2,3-dimethyl-5,6,7,8-

tetrahydrofuro[2,3-b]quinolin-4-yl)acetoamide on deficits of water maze learning in rats. Arzneim.-Forsch/Drug Res 1996; 46(1): 369373.

16. Takashina K, Bessho T, Mori R,EguchiJ, Saito K. MKC-231, a choline uptake enhancer: (2) Effect on synthesisand release of acetylcholine in AF64A-treated rats. J Neural Transm2008; 115: 1-27-1035. 